\title{
Multimode extensions of Combinatorial Optimization problems.
}

\author{
Roberto Cordone $^{1}$ \\ University of Milano, Department of Computer Science, via Comelico 39, 20135 \\ Milano, Italy \\ Guglielmo Lulli ${ }^{2}$ \\ Lancaster University Management School, LA1 4YX Lancaster, UK
}

Keywords: Combinatorial Optimization, covering problems, complexity, Variable Neighborhood Search.

\section{Introduction}

Motivated by different real life applications, some combinatorial optimization problems have been generalized to the multimode setting. A primer in this category is the well-known Resource Constrained Project Scheduling problem (RCPSP) [1], which consists in determining the starting times of all the activities of a project in order to minimize the total completion time of the project. The multimode extension considers the case where each activity may be executed in one out of a set $M$ of modes.

Recently, we have been investigating the multimode setting in other combinatorial optimization problems, e.g., the Set Covering Problem (SCP) [2] and the Covering Location Problem (CLP) [3]. The former is the combination of $|M|$ single mode SCP instances, defined on the same ground set $I$, which

1 Email: roberto.cordone@unimi.it

2 Email: g.lulli@lancaster.ac.uk 
must be covered in all modes of $M$. The single mode subproblems are linked by cardinality constraints, which impose a limit on the number of modes in which the same column from $J$ can be included in the solution.

The generalization of the CLP to the multimode setting consists in placing a given number $K_{m}$ of facilities of each mode $m \in M$ to serve a set $I$ of demand centers that require different types of service. The goal is to maximize the demand coverage over all centers and modes with a cardinality constraint that limits the number of different modes activated in each facility site.

The decision version of all the multimode problems listed above, i.e., RCPSP, SCP and CLP, are $\mathcal{N} \mathcal{P}$-complete because they include, as a special case, the corresponding single mode version. However, the introduction of additional modes makes them much more challenging to solve. The higher level of complexity does not exclusively refer to the worst case analysis (i.e., computational complexity and approximability), but indeed also to the average case (i.e., exact and heuristic algorithms).

In what follows, we focus on the SCP and CLP. We first review some complexity results for their multimode generalizations and then present the computational challenges and a viable heuristic approach based on the Variable Neighborhood Search (VNS) framework.

\section{Complexity results}

\section{Set covering}

The single mode SCP admits a logarithmic approximation guarantee and approximation results are also available for more general covering problems [4]. By contrast, even the feasibility of the multimode SCP is $\mathcal{N} \mathcal{P}$-complete.

Theorem 2.1 It is $\mathcal{N} \mathcal{P}$-complete to determine whether a given instance of the multimode $S C P$ is feasible or not, even if $|M|=2$.

With the same construction it is possible to prove the inapproximability.

Corollary 2.2 The MM-SCP does not admit any polynomial algorithm with an approximation guarantee, unless $\mathcal{P}=\mathcal{N} \mathcal{P}$.

\section{Facility location}

The CLP has a constant approximation guarantee, as discussed in Vohra and Hall [5]. Under mild technical assumptions, we provide two greedy algorithms that compute feasible solutions with a guaranteed approximation for the multimode CLP. Algorithm Greedy1 selects one column at a time which covers 
the uncovered set of rows of maximum weight, satisfying the cardinality constraint. Algorithm Greedy2 first builds a solution like Greedy1, but relaxing the cardinality constraints, then retrieves a feasible solution by removing the facilities which leave uncovered the minimum weight set of rows as necessary.

Theorem 2.3 Algorithm Greedy1 computes a solution of MM-CLP with a guaranteed approximation factor of

$$
\alpha_{1}=\frac{\sum_{m \in M} K_{m} W_{m}}{|J| W_{\text {tot }}}
$$

where $W_{m}=\sum_{i \in I} w_{i m}$ is the total weight of all rows in mode $m \in M$ and $W_{\text {tot }}=\sum_{m \in M} W_{m}$ is the total weight of all rows in all modes.

When all modes have the same total weight $\left(W_{m}=W\right)$ and require the same number of facilities $\left(K_{m}=K\right), \alpha_{1}=K /|J|$. If all columns can be selected in one single mode $\left(b_{j}=1\right)$, the approximation can be refined.

Corollary 2.4 If $K_{m}=K$ and $W_{m}=W$ for all $m \in M$, and $b_{j}=1$ for all $j \in J$, Algorithm Greedy1 provides a constant approximation factor equal to

$$
\alpha_{1}^{\prime}=\frac{K}{|J|}\left(\frac{1}{|M|}+\frac{|J|}{K|M|} \ln \frac{1}{1-\frac{K}{|J|}(|M|-1)}\right)
$$

Theorem 2.5 Algorithm Greedy2 computes a solution of MM-CLP with a guaranteed approximation factor of

$$
\alpha_{2}=\frac{b_{\min }}{|M|}\left[1-\left(1-\frac{1}{K_{\min }}\right)^{K_{\min }}\right]
$$

where $b_{\min }=\min _{j \in J} b_{j}$ and $K_{\min }=\min _{m \in M} K_{m}$.

In Table 1, we summarize the complexity results described so far.

\section{Computational experience and heuristic approach}

Although in terms of computational complexity the mentioned problems are all NP-hard, the multimode version experimentally proves much harder. Indeed, whilst state-of-the-art ILP solvers like CPLEX are able to quickly solve to optimality average-sized instances of the single mode versions, the same is not true for the multimode instances. Our computational experience shows that 


\begin{tabular}{c|c|c} 
& Single-mode & Multi-mode \\
\hline \multirow{3}{*}{ SCP } & Feas. easy & Feas. NP-complete \\
& NP-hard & NP-hard \\
& log-APX & not APX \\
\hline MCLP & NP-hard & NP-hard \\
& APX with $\alpha_{s m}$ & APX with $\alpha_{m m} \approx \alpha_{s m} /|M|$
\end{tabular}

Table 1

Comparison of complexity between singlemode and multimode problems

the ILP solver is unable to close the gap in hours of computation on instances of a few thousands variables and constraints. More specifically, the average gap for the CLP is always around 10\%, whereas the situation is even gloomier for the SCP, as the ILP in some cases is unable to find a feasible solution.

Given this experience, we developed a metaheuristic approach based on the VNS framework. We will discuss neighborhoods of different typologies and exploration strategies (exchange mechanisms), which are very promising to solve multimode problems. Computational results show that it is possible to achieve in a matter of minutes a $5 \%$ gap with respect to the known bound provided by the solver (which is unlikely to be tight).

\section{References}

[1] C. Artigues, S. Demassey and E. Néron (eds.) Resource-Constrained Project Scheduling: Models, Algorithms, Extensions and Applications. Wiley (2008)

[2] F. Colombo, R. Cordone and G. Lulli (2015). A Variable Neighborhood Search algorithm for the Multimode Set Covering Problem, J. of Global Optimization 65 (3) pp. 461-480.

[3] F. Colombo, R. Cordone and G. Lulli (2016). The Multimode Covering Location Problem, Computers \& Operations Research 67 pp. 25-33.

[4] A. Srinivasan (2006) An Extension of the Lovász Local Lemma, and its Applications to Integer Programming. SIAM Journal on Computing, 36 (3), pp. 609-634.

[5] R.V. Vohra, N. Hall (1993) A probabilistic analysis of the maximal covering location problem Discrete Applied Mathematics, 43, pp. 175-183. 